\title{
Tratamento da compactação experimental do cólon maior em eqüinos: resultados de laboratório e exames bioquímicos
}

[Treatment of experimental impaction in equine large colon: laboratory findings and biochemical test]

\author{
G.E.S. Alves ${ }^{1}$, J.D. Ribeiro Filho ${ }^{2}$, H.P. Oliveira ${ }^{1}$, J.M.G. Abreu ${ }^{3}$ \\ ${ }^{1}$ Escola de Veterinária - Universidade Federal de Minas Gerais \\ Caixa Postal 567 \\ 30123-970 - Belo Horizonte, MG \\ ${ }^{2}$ Departamento de Veterinária - Universidade Federal de Viçosa - Viçosa, MG \\ ${ }^{3}$ Universidade Estadual do Ceará - Fortaleza, CE
}

\begin{abstract}
RESUMO
Avaliou-se ação da solução isotônica poliônica enteral da sene mais fluidoterapia intravenosa e da fluidoterapia intravenosa no tratamento da compactação no cólon maior em eqüinos. Foram utilizados 20 animais divididos em quatro grupos. Os animais dos grupos tratados eram portadores de compactação no cólon maior induzida experimentalmente. O grupo C, controle, não foi tratado, o grupo E8 foi tratado com solução isotônica poliônica enteral ( $8 \mathrm{ml} / \mathrm{kg} / \mathrm{hora} / 48$ horas), o grupo SE recebeu sene (20mg/kg, duas doses de $24 / 24 \mathrm{~h}$ ) mais ringer lactato intravenoso (10 $\mathrm{ml} / \mathrm{kg} / \mathrm{hora} / 12$ horas, durante dois dias) e o grupo RL recebeu ringer lactato intravenoso $(16 \mathrm{ml} / \mathrm{kg} / \mathrm{hora} / 12 \mathrm{horas}$, durante dois dias). O tratamento E8 provocou hipernatremia, hipercloremia e hiperglicemia. No grupo SE foi observada maior concentração dos valores de uréia, creatinina, osmolalidade e lactato plasmático. $\mathrm{O}$ tratamento $\mathrm{RL}$ não resultou em alteração no equilíbrio hidroeletrolítico e foi o mais eficiente para a correção dos valores bioquímicos em eqüinos com compactação do cólon maior, seguido pelo E8. O SE foi o menos eficiente na normalização das variáveis bioquímicas.
\end{abstract}

Palavras-chave: eqüino, compactação, cólon maior, fluidoterapia, laxantes

\begin{abstract}
The effects of isotonic polionic enteral solution, sene plus intravenous (IV) fluid therapy or fluid therapy $I V$ only for the large colon impaction treatment in horses were evaluated. Twenty animals were divided in four groups. Animals of the treated groups had experimentally induced colon impactions. Group C (control) was not treated; group E8 received isotonic polionic enteral solution $(8 \mathrm{ml} / \mathrm{kg} / \mathrm{hour} / 48$ hours); group SE received sene $(20 \mathrm{mg} / \mathrm{kg}$, two doses with 24 hours interval) and ringer lactate IV $(10 \mathrm{ml} / \mathrm{kg} / \mathrm{hour} / 12$ hour, during two days), and group $R L$ received ringer lactate $\mathrm{IV}(16 \mathrm{ml} / \mathrm{kg} / \mathrm{hour} / 12 \mathrm{hour}$, during two days) only. Animals in the E8 group had hypernatremia, hypercloremia and hyperglycemia. Animals of the SE group had higher concentration of urea, creatinine, osmolality and plasmatic lactate. Ringer lactate IV only, group RL, did not cause alteration in the electrolytic equilibrium. In conclusion, intravenous fluid therapy with Ringer solution alone was the most efficient treatment for biochemical correction in horses with large colon impaction. Fluid therapy was also efficient in minor degree. Sene treatment associated with intravenous fluid therapy had the poorest results among the protocols to normalize biochemical variables.
\end{abstract}

Keywords: equine, impaction, large colon, fluid therapy, laxatives

Recebido para publicação em 23 de abril de 2004

Recebido para publicação, após modificações, em 4 de outubro de 2004

E-mail: geraldo@vet.ufmg.br 


\section{INTRODUÇÃO}

As obstruções simples do sistema digestório em eqüinos são causas importantes da síndrome cólica. Segundo White (1990), elas representam $35,3 \%$ dos casos de cólica, sendo o cólon maior o segmento afetado em $28,6 \%$ dos casos. As obstruções simples, denominadas compactação, apresentam incidência elevada e ocorrem em qualquer segmento intestinal, mas são, particularmente, freqüentes no intestino grosso, sobretudo no cólon maior (Dabareiner e White, 1995; White e Dabareiner, 1997). A compactação do cólon maior pode ser dividida em duas categorias, organizada e nãoorganizada. Embora clinicamente sejam semelhantes em ambas as categorias, a forma não-organizada é a causa mais comum de cólica e, na maioria dos casos, os pacientes afetados recuperam-se apenas com a terapia medicamentosa (Doran, 1993), constituída de controle da dor, hidratação intravenosa ou oral, e administração de laxantes (Sullins, 1990; Dabareiner e White, 1995).

Os laxantes à base de sene estão entre os mais utilizados. As plantas sene são pequenos arbustos da família das leguminosas. As mais utilizadas são a Cassia acutifolia Delite e a Cassia augustifolia Vahl, comumente conhecidas como sene-alexandria e tinevelly, respectivamente. Os componentes da planta utilizados como matériaprima são as folhas secas e as vagens (Franz, 1993). A sene está classificada entre os laxativos antranóides, derivados das antraquinonas, e tem como princípio ativo os senosídeos A e B. São farmacologicamente inativos, comportando-se como pró-drogas naturais. No cólon maior os senosídeos são metabolizados pelas enzimas bacterianas da microbiota intestinal, originando a reinantrona, metabólito ativo responsável pelo efeito laxante (Lemli, 1988; Yagi et al., 1991).

Embora o intestino grosso do eqüino seja um órgão anatômico e funcionalmente de relativa complexidade, empregam-se como laxativos, freqüentemente, o sulfato de magnésio e o óleo mineral, ambos com vantagens, efeitos colaterais e limitações (Freeman et al., 1992). Pela importância clínica e prevalência da compactação do cólon maior e pela escassez de estudos controlados utilizando laxantes em eqüinos portadores de compactação do cólon, este estudo teve o objetivo de avaliar o efeito da fluidoterapia enteral, intravenosa e do laxativo sene em eqüinos com compactação experimental do cólon maior sob variáveis bioquímicas.

\section{MATERIAL E MÉTODOS}

Utilizaram-se 20 eqüinos, quatro fêmeas (nãogestantes) e 16 machos (quatro não-castrados e 12 castrados), sem raça definida, com idades entre três e 22 anos, escore corporal de três a quatro (Speirs, 1997) e peso corporal médio de $295,52 \mathrm{~kg}$. Uma semana antes do experimento, após avaliação clínica, fez-se o controle de endoparasitas (febendazole ${ }^{1}-7,2 \mathrm{mg} / \mathrm{kg}$ ) e ectoparasitas (deltametrina ${ }^{2}$ a $0,025 \%$ ). Os animais foram alojados em cocheiras individuais para adaptação à dieta com água e feno de coast cross (Cynodon dactylon) à vontade. A ração concentrada comercial ${ }^{3}$ foi fornecida duas vezes ao dia em quantidade equivalente a $1 \%$ do peso corpóreo, adicionada de $50 \mathrm{~g} / \mathrm{dia}$ de suplemento mineral ${ }^{4}$. Utilizou-se o modelo de compactação no cólon maior, protocolo III, de Ribeiro Filho e Alves (2002).

Os eqüinos foram distribuídos aleatoriamente em quatro grupos de cinco animais (uma fêmea e quatro machos) - um grupo-controle (sem indução de compactação e sem tratamento) e três grupos tratados, de modo uniforme em relação à idade, ao sexo e ao escore corporal. Ao final das $72 \mathrm{~h}$ da fase de indução da compactação, após confirmar a compactação do cólon maior por exame transretal, iniciaram-se os tratamentos: $o$ grupo E8 foi tratado com solução isotônica poliônica enteral $\left(\mathrm{NaCl} 5 \mathrm{~g}+\mathrm{KCl} 1 \mathrm{~g}+\mathrm{NaHCO}_{3}\right.$ $4 \mathrm{~g}+$ glicose $5 \mathrm{~g}$ ) para completar o volume de $1000 \mathrm{ml}$, administrados em bolus na dose de $8 \mathrm{ml} / \mathrm{kg} / \mathrm{h} / 48 \mathrm{~h}$ da solução via sonda nasogástrica; o grupo SE recebeu solução isotônica parenteral intravenosa de Ringer lactato ${ }^{5}(10 \mathrm{ml} / \mathrm{kg} / \mathrm{h} / 12 \mathrm{~h})$ associada à sene na dose $20 \mathrm{mg} / \mathrm{kg}$ (uma vez ao dia) diluídos em 1000ml de água, nos dias 1 e 2 de tratamento; o grupo RL recebeu solução isotônica parenteral intravenosa de Ringer lactato $^{5}(16 \mathrm{ml} / \mathrm{kg} / \mathrm{h} / 12 \mathrm{~h})$, nos dias 1 e 2 de tratamento.

\footnotetext{
${ }^{1}$ Panacur pasta - Intervet S. A. - São Paulo-SP

${ }^{2}$ Butox P - Intervet S. A. - São Paulo-SP

${ }^{3}$ Nutriage - Guabi S. A. - Além Paraíba-MG

${ }^{4}$ Centauro - Guabi S. A. - Além Paraíba-MG

${ }^{5}$ Fresenius Kabi Brasil Ltda - Campinas-SP
} 
Procedeu-se à avaliação laboratorial das proteínas plasmáticas totais ${ }^{6}$, sódio ${ }^{7}$, potássio ${ }^{7}$, cloreto $^{8}$, uréia $^{8}$, creatinina ${ }^{8}$, magnésio total ${ }^{8}$, osmolalidade sérica ${ }^{9}$, glicose $^{10}$ e lactato $^{10}$, segundo os intervalos: T0i, T24i, T48i e T72i (correspondente à fase de indução da compactação), T12t, T24t e T48t (correspondente à fase de tratamento) e T72f (correspondente à fase de avaliação final).

As variáveis foram submetidas ao teste de normalidade e homocedasticidade. Os dados foram submetidos à analise de variância com base em um planejamento de medidas repetidas, ou seja, cada tratamento foi avaliado em vários momentos do acompanhamento, usando-se o programa SAS (User's..., 1985). Avaliaram-se os efeitos do tratamento, do tempo e a interação tratamento $\times$ tempo. Ao apresentarem significância, as variáveis foram submetidas ao teste de comparações múltiplas de médias LSD. Quando as variáveis não atenderam às premissas, mesmo após as transformações, elas foram avaliadas pelos testes não paramétricos de Kruskal-Wallis (entre tratamentos) e Friedman (tratamento ao longo do tempo) (Conover, 1980). Todos os resultados foram considerados significativos quando $\mathrm{P}<0,05$.

\section{RESULTADOS E DISCUSSÃO}

Observou-se aumento (Tab. 1) na concentração de proteínas plasmáticas totais (PPT) ao longo da fase de indução da compactação (T0i a T72i) nos grupos $\mathrm{E} 8, \mathrm{SE}$ e $\mathrm{RL}(\mathrm{P}=0,0001)$, ocasionado pela desidratação que se instalou nos animais durante essa fase, confirmando os resultados de Dabareiner e White (1995) e Ribeiro Filho e Alves (2002). Na fase subseqüente, como resultado da soroterapia, a concentração das PPT diminui nos grupos E8, SE e RL. Resultado importante foi observado no T12t, em que os grupos $\mathrm{SE}$ e RL, após 12 horas de soroterapia intravenosa, apresentaram valores menores de PPT que o grupo E8, no qual foi administrada soroterapia via oral $(\mathrm{P}=0,0003)$. Nos grupos $\mathrm{SE}$ e RL, no T12t a concentração de PPT foi semelhante à do $\mathrm{T} 0 \mathrm{i}$, enquanto que no grupo $\mathrm{E} 8$

\footnotetext{
${ }^{6}$ Refratômetro SPR T2 - Atago

${ }^{7}$ Fotômetro de chama F250 - CELM

${ }^{8}$ Cobas - Roche

${ }^{9}$ Vapor Presure Osmometer 5520 - Claremont - Canada

${ }^{10}$ Espectrofotômetro E 225D - CELM
}

apenas no T48t a concentração foi semelhante à do T0i. Esses resultados demonstraram que a soroterapia intravenosa foi mais eficiente na expansão da volemia do que a enteral.

Durante a fase de indução da compactação (T0i a T72i), houve aumento gradual do teor do sódio sérico nos animais dos grupos E8, SE e RL $(\mathrm{P}=0,0001)$, ocasionado pela desidratação. Esses resultados confirmam os de Genetzky et al. (1987), que submeteram eqüinos a jejum hídrico por 72 horas para avaliar alterações laboratoriais. Dabareiner e White (1995), ao avaliarem eqüinos com compactação do cólon maior, não encontraram alterações, apesar de os pacientes terem apresentado desidratação. Os autores descreveram animais com até quatro segundos de tempo de enchimento capilar e hematócrito de $61 \%$, o que caracterizou desidratação de moderada a intensa. Neste trabalho, após o início do tratamento nos animais dos grupos SE e RL, houve declínio gradual do sódio em conseqüência da correção da desidratação dos pacientes com solução poliônica via intravenosa $(\mathrm{P}=0,0001)$.

No grupo E8 ocorreu aumento nos valores do sódio sérico (hipernatremia) após o início do tratamento. Deve-se considerar como causa a formulação da solução enteral utilizada que, apesar de ser isotônica, apresentou concentrações de sódio e cloreto maiores que as do plasma, sobrevindo hipernatremia e hipercloremia após sua administração. Associado a esse fato, em dois animais foi observado valor elevado de sódio no T0i $(151 \mathrm{mmol} / 1)$, durante as fases de indução e tratamento. $\mathrm{Na}$ avaliação final (T72f), eles continuaram a ser os maiores valores, o que favoreceu a elevação da média do grupo. Esse resultado sugere anormalidade no mecanismo de excreção do sódio, através dos rins, sinalizando a possibilidade de doença renal como citou DiBartola (1992). Neste estudo não foi realizada a avaliação laboratorial da urina. Segundo Johnson (1995), para o entendimento correto da concentração anormal de sódio sérico, é necessária também a avaliação da sua taxa de excreção pelos rins. 
Tabela 1. Proteínas plasmáticas totais $(\mathrm{g} / \mathrm{dl})$, sódio $(\mathrm{mmol} / \mathrm{l})$, cloreto $(\mathrm{mmol} / \mathrm{l})$, potássio $(\mathrm{mmol} / \mathrm{l})$ e osmolalidade sérica $(\mathrm{mmol} / \mathrm{l})$ de eqüinos submetidos a diferentes tratamentos da compactação experimental no cólon maior

\begin{tabular}{|c|c|c|c|c|c|c|c|c|}
\hline Tratamento & T0i & $\mathrm{T} 24 \mathrm{i}$ & $\mathrm{T} 48 \mathrm{i}$ & $\mathrm{T} 72 \mathrm{i}$ & $\mathrm{T} 12 \mathrm{t}$ & $\mathrm{T} 24 \mathrm{t}$ & $\mathrm{T} 48 \mathrm{t}$ & $\mathrm{T} 72 \mathrm{f}$ \\
\hline \multicolumn{9}{|c|}{ Proteínas plasmáticas totais ${ }^{\mathrm{a}}$ (média \pm desvio-padrão) } \\
\hline $\mathrm{C}$ & $7,3 \pm 0,3$ & $7,3 \pm 0,3$ & $7,4 \pm 0,2$ & $7,2 \pm 0,1$ & $7,4 \pm 0,4$ & $7,2 \pm 0,2$ & $7,3 \pm 0,3$ & $7,2 \pm 0,4$ \\
\hline E8 & $7,5 \pm 0,3$ & $8,9 \pm 0,2$ & $9,4 \pm 0,4$ & $11,0 \pm 0,6$ & $8,9 \pm 0,5$ & $8,0 \pm 0,0$ & $7,7 \pm 0,3$ & $7,6 \pm 0,2$ \\
\hline SE & $7,0 \pm 0,4$ & $8,5 \pm 0,4$ & $8,8 \pm 0,3$ & $9,7 \pm 0,8$ & $7,6 \pm 0,3$ & $8,0 \pm 0,6$ & $7,2 \pm 0,8$ & $6,8 \pm 0,2$ \\
\hline \multirow[t]{2}{*}{$\mathrm{RL}$} & $7,5 \pm 0,4$ & $9,1 \pm 0,8$ & $9,0 \pm 0,9$ & $10,0 \pm 1,3$ & $7,3 \pm 0,6$ & $8,0 \pm 0,9$ & $7,5 \pm 0,6$ & $7,2 \pm 0,4$ \\
\hline & $\mathrm{C}=\mathrm{E} 8=\mathrm{SE}=\mathrm{RL}$ & $\mathrm{E} 8=\mathrm{SE}=\mathrm{RL}>\mathrm{C}$ & $\mathrm{E} 8=\mathrm{SE}=\mathrm{RL}>\mathrm{C}$ & $\mathrm{E} 8>\mathrm{SE}=\mathrm{RL}>\mathrm{C}$ & $\mathrm{E} 8>\mathrm{C}=\mathrm{SE}=\mathrm{RL}$ & $\mathrm{E} 8=\mathrm{SE}=\mathrm{RL}>\mathrm{C}$ & $\mathrm{C}=\mathrm{E} 8=\mathrm{SE}=\mathrm{RL}$ & $\mathrm{E} 8>\mathrm{SE}$ \\
\hline \multicolumn{9}{|c|}{ Sódio a (média+desvio-padrão) } \\
\hline $\mathrm{C}$ & $144,4 \pm 3,1$ & $141,2 \pm 3,4$ & $144,2 \pm 4,0$ & $143,4 \pm 4,3$ & $144,0 \pm 4,2$ & $145,0 \pm 4,6$ & $142,8 \pm 2,9$ & $140,0 \pm 3,0$ \\
\hline E8 & $148,2 \pm 3,9$ & $166,4 \pm 6,7$ & $164,2 \pm 13,6$ & $162,4 \pm 10,5$ & $174,2 \pm 8,9$ & $172,4 \pm 13,2$ & $165,0 \pm 9,9$ & $155,0 \pm 3,6$ \\
\hline SE & $137,3 \pm 7,1$ & $147,5 \pm 8,7$ & $148,3 \pm 15,1$ & $157,0 \pm 20,8$ & $142,5 \pm 6,4$ & $152,0 \pm 5,0$ & $133,5 \pm 9,9$ & $131,8 \pm 7,7$ \\
\hline \multirow[t]{2}{*}{$\mathrm{RL}$} & $138,4 \pm 2,9$ & $142,0 \pm 4,1$ & $151,6 \pm 5,1$ & $150,0 \pm 9,1$ & $142,0 \pm 5,2$ & $143,8 \pm 10,9$ & $134,8 \pm 8,6$ & $136,6 \pm 4,7$ \\
\hline & $\mathrm{C}=\mathrm{E} 8=\mathrm{SE}=\mathrm{RL}$ & $\mathrm{E} 8>\mathrm{C}=\mathrm{SE}=\mathrm{RL}$ & $\mathrm{E} 8>\mathrm{C}=\mathrm{SE}=\mathrm{RL}$ & $\mathrm{E} 8>\mathrm{RL}, \mathrm{C}$ SE $>\mathrm{C}$ & $\mathrm{E} 8>\mathrm{RL}=\mathrm{SE}=\mathrm{C}$ & $\mathrm{E} 8>\mathrm{RL}=\mathrm{SE}=\mathrm{C}$ & $\mathrm{E} 8>\mathrm{RL}=\mathrm{SE}=\mathrm{C}$ & $\mathrm{E} 8>\mathrm{RL}=\mathrm{SE}=\mathrm{C}$ \\
\hline \multicolumn{9}{|c|}{ Osmolalidade sérica $^{\text {a }}($ média \pm desvio-padrão) } \\
\hline $\mathrm{C}$ & $276,6 \pm 6,2$ & $269,6 \pm 9,5$ & $270,2 \pm 2,4$ & $271,8 \pm 6,5$ & $275,8 \pm 4,8$ & $275,6 \pm 0,6$ & $276,4 \pm 3,1$ & $276,2 \pm 7,1$ \\
\hline E8 & $251,0 \pm 9,3$ & $266,4 \pm 11,9$ & $275,6 \pm 10,7$ & $316,0 \pm 42,9$ & $313,0 \pm 12,3$ & $291,6 \pm 17,6$ & $269,0 \pm 9,5$ & $250,6 \pm 4,7$ \\
\hline SE & $270,5 \pm 4,5$ & $302,0 \pm 12,4$ & $320,5 \pm 18,5$ & $352,0 \pm 30,8$ & $316,3 \pm 23,6$ & $338,8 \pm 28,0$ & $333,8 \pm 6,7$ & $346,3 \pm 7,4$ \\
\hline \multirow[t]{2}{*}{$\mathrm{RL}$} & $266,0 \pm 12,2$ & $286,8 \pm 8,1$ & $301,2 \pm 17,3$ & $317,4 \pm 29,8$ & $284,4 \pm 25,7$ & $281,4 \pm 19,5$ & $249,6 \pm 8,9$ & $246,6 \pm 3,7$ \\
\hline & $\mathrm{C}=\mathrm{E} 8=\mathrm{SE}=\mathrm{RL}$ & $\mathrm{SE}>\mathrm{C}=\mathrm{E} 8$ & $\mathrm{SE}=\mathrm{RL}>\mathrm{E} 8=\mathrm{C}$ & $\mathrm{SE}>\mathrm{RL}=\mathrm{E} 8>\mathrm{C}$ & $\mathrm{E} 8=\mathrm{SE}>\mathrm{RL}=\mathrm{C}$ & $\mathrm{SE}>\mathrm{C}=\mathrm{E} 8=\mathrm{RL}$ & $\mathrm{SE}>\mathrm{C}, \mathrm{E} 8, \mathrm{RL} \mathrm{C}>\mathrm{RL}$ & $\mathrm{SE}>\mathrm{C}>\mathrm{E} 8=\mathrm{RL}$ \\
\hline \multicolumn{9}{|c|}{ Potássio ${ }^{\text {a }}$ (média+desvio-padrão) } \\
\hline $\mathrm{C}$ & $4,3 \pm 0,5$ & $4,3 \pm 0,5$ & $4,3 \pm 0,2$ & $4,2 \pm 0,5$ & $4,0 \pm 0,4$ & $4,6 \pm 0,5$ & $4,3 \pm 0,3$ & $4,3 \pm 0,4$ \\
\hline E8 & $4,4 \pm 0,4$ & $4,2 \pm 0,3$ & $4,7 \pm 0,8$ & $4,6 \pm 0,5$ & $4,3 \pm 0,2$ & $3,3 \pm 0,4$ & $3,0 \pm 0,7$ & $3,5 \pm 1,2$ \\
\hline SE & $4,3 \pm 0,1$ & $3,9 \pm 0,3$ & $4,0 \pm 0,8$ & $5,1 \pm 0,6$ & $4,1 \pm 0,8$ & $3,6 \pm 0,6$ & $3,3 \pm 0,7$ & $3,3 \pm 0,8$ \\
\hline \multirow[t]{2}{*}{$\mathrm{RL}$} & $4,3 \pm 0,3$ & $3,6 \pm 0,2$ & $4,4 \pm 0,3$ & $4,2 \pm 0,4$ & $3,9 \pm 0,4$ & $3,5 \pm 0,6$ & $4,1 \pm 0,6$ & $4,1 \pm 0,9$ \\
\hline & $\mathrm{C}=\mathrm{E} 8=\mathrm{SE}=\mathrm{RL}$ & $\mathrm{C}=\mathrm{E} 8=\mathrm{SE}=\mathrm{RL}$ & $\mathrm{C}=\mathrm{E} 8=\mathrm{SE}=\mathrm{RL}$ & $\mathrm{SE}>\mathrm{C}=\mathrm{RL}$ & $\mathrm{C}=\mathrm{E} 8=\mathrm{SE}=\mathrm{RL}$ & $\mathrm{C}>\mathrm{E} 8=\mathrm{SE}=\mathrm{RL}$ & $\mathrm{C}=\mathrm{RL}>\mathrm{E} 8=\mathrm{SE}$ & $\mathrm{C}=\mathrm{RL}>\mathrm{E} 8=\mathrm{SE}$ \\
\hline \multicolumn{9}{|c|}{ Cloreto $^{\mathrm{b}}($ média \pm desvio-padrão) } \\
\hline $\mathrm{C}$ & $106,0 \pm 5,0$ & $103,9 \pm 3,4$ & $104,9 \pm 2,1$ & $104,0 \pm 5,4$ & $108,0 \pm 3,7$ & $104,6 \pm 4,4$ & $103,1 \pm 4,9$ & $104,7 \pm 3,1$ \\
\hline E8 & $106,0 \pm 7,1$ & $100,6 \pm 13,7$ & $113,6 \pm 11,0$ & $115,7 \pm 9,9$ & $112,1 \pm 12,9$ & $121,2 \pm 14,1$ & $102,1 \pm 9,4$ & $96,3 \pm 9,0$ \\
\hline SE & $98,2 \pm 6,1$ & $95,8 \pm 9,3$ & $101,0 \pm 9,5$ & $96,3 \pm 11,7$ & $102,1 \pm 10,6$ & $102,6 \pm 13,0$ & $95,7 \pm 8,1$ & $95,1 \pm 7,7$ \\
\hline \multirow[t]{2}{*}{$\mathrm{RL}$} & $100,1 \pm 3,1$ & $93,5 \pm 7,1$ & $97,0 \pm 10,4$ & $91,4 \pm 14,4$ & $102,1 \pm 16,5$ & $102,8 \pm 11,6$ & $94,2 \pm 10,5$ & $98,5 \pm 8,3$ \\
\hline & $\mathrm{C}=\mathrm{E} 8=\mathrm{SE}=\mathrm{RL}$ & $\mathrm{C}=\mathrm{E} 8=\mathrm{SE}=\mathrm{RL}$ & $\mathrm{C}=\mathrm{E} 8=\mathrm{SE}=\mathrm{RL}$ & $\mathrm{E} 8>\mathrm{SE}=\mathrm{RL}$ & $\mathrm{C}=\mathrm{E} 8=\mathrm{SE}=\mathrm{RL}$ & $*$ & $*$ & $*$ \\
\hline
\end{tabular}

T0i-T72i: período de indução de compactação.

T12t-T48t: período de tratamento.

T72t: período final de tratamento.

${ }^{\mathrm{a}}$ Análise de variância (medidas repetidas); ${ }^{\mathrm{b}}$ Kruskal-Wallis (entre tratamentos) e Friedman (tratamento ao longo do tempo; *Nos tempos $24 \mathrm{t}$, $48 \mathrm{t}$ e $72 \mathrm{f}$ os tratamentos não foram comparados, pois um animal do grupo SE morreu.

$\mathrm{C}=$ controle; $\mathrm{E} 8=$ solução isotônica poliônica enteral $\left(\mathrm{NaCl} 5 \mathrm{~g}+\mathrm{KCl} 1 \mathrm{~g}+\mathrm{NaHCO}_{3} 4 \mathrm{~g}+\mathrm{glicose} 5 \mathrm{~g}-\mathrm{qsq} 1000 \mathrm{ml}\right), \mathrm{dose}$ de $8 \mathrm{ml} / \mathrm{kg} / \mathrm{h} / 48 \mathrm{~h} \mathrm{na}$ sonda nasogástrica; $\mathrm{SE}=$ solução isotônica intravenosa de Ringer lactato $(10 \mathrm{ml} / \mathrm{kg} / \mathrm{h} / 12 \mathrm{~h})$ associada à sene, dose de $20 \mathrm{mg} / \mathrm{kg} ; \mathrm{RL}=\mathrm{solução} \mathrm{isotônica} \mathrm{de}$ Ringer lactato $(16 \mathrm{ml} / \mathrm{kg} / \mathrm{h} / 12 \mathrm{~h})$.

O teor sérico do cloreto na fase de indução da compactação apresentou discreta diminuição nas primeiras 24 horas (T24i) nos grupos E8, SE e RL, ocasionada pela administração da furosemida, como descrito por Freestone et al. (1989). No grupo E8, após discreta diminuição no T24i, em T48i e T72i houve aumento gradual na concentração do cloreto. Como aconteceu com o sódio, esse aumento foi ocasionado pela desidratação $(\mathrm{P}=0,019)$. Nos grupos $\mathrm{SE}$ e RL no T72i ocorreu discreta diminuição nos valores do cloreto, o que não se associa com a ação da furosemida, pois já haviam transcorrido 36 horas após a última administração. Segundo Johnson (1995), usualmente hipocloremia pode ocorrer em associação com aumento do ânion gap ou como parte da resposta compensatória à acidose respiratória. Após o início do tratamento, houve aumento nos teores séricos do cloreto nos animais dos grupos E8, (T24t), SE e RL (T12t) em efeito à administração da solução enteral (grupo E8) e do ringer lactato (grupos SE e RL). No T48t e T72f ocorreu diminuição na concentração de cloreto nos animais do grupo E8 em conseqüência de o volume plasmático ter sido restabelecido, normalizando a função renal.

Diminuição discreta, nas primeiras 24 horas (T24i) da fase de indução da compactação, foi observada nos valores do potássio nos grupos E8, SE e RL em função do mecanismo já descrito para o cloreto. Em seguida, observou-se aumento nos valores até o final da fase de indução da compactação (T72i), ocasionado pela desidratação. Associado a esse fato, a presença da acidose metabólica, decorrente da hipovolemia, também contribuiu para o aumento do potássio, como descreveu Kasari (1999). A diminuição gradual na concentração do potássio dos grupos E8, SE e RL, observada a partir do $\mathrm{T} 12 \mathrm{t}$, originou-se da expansão do volume plasmático sobrevinda da fluidoterapia e da 
migração do potássio do líquido extracelular para o intracelular, evento que ocorre quando há correção da acidose metabólica.

A osmolalidade sérica nos grupos E8, SE e RL elevou-se gradualmente durante a fase de indução da compactação, atingindo no T72i os maiores valores $(\mathrm{P}=0,0001)$, confirmando a presença de desidratação. Deve-se considerar que, além do sódio e do cloreto, potássio, glicose e, principalmente, uréia ou algum metabólito osmoticamente ativo não mensurado podem ter sido os responsáveis pelo aumento da osmolalidade, como relatou Gennari (1984).
Como a glicose e a uréia (Tab. 2) apresentaram valores elevados durante esse período, presumese que elas também tiveram participação no aumento da osmolalidade na fase de indução. A partir de $\mathrm{T} 12 \mathrm{t}$, houve diminuição gradual dos valores da osmolalidade decorrente da expansão do volume plasmático pela soroterapia nos grupos E8 e RL. O grupo SE, após discreta diminuição no $\mathrm{T} 12 \mathrm{t}$, voltou a apresentar aumento da osmolalidade em T24t, T48t e T72f (Tab. 1). Percebem-se no grupo SE valores elevados na concentração da uréia nos tempos 12t, 24t, 48t e $72 \mathrm{f}$, justificando os resultados obtidos.

Tabela 2. Glicose (mg/dl), uréia (mg/dl), creatinina (mg/dl), lactato (mmol/l) e magnésio total (mmol/l) de eqüinos submetidos a diferentes tratamentos da compactação experimental no cólon maior

\begin{tabular}{|c|c|c|c|c|c|c|c|c|}
\hline Tratamento & T0i & $\mathrm{T} 24 \mathrm{i}$ & $\mathrm{T} 48 \mathrm{i}$ & $\mathrm{T} 72 \mathrm{i}$ & $\mathrm{T} 12 \mathrm{t}$ & $\mathrm{T} 24 \mathrm{t}$ & $\mathrm{T} 48 \mathrm{t}$ & $\mathrm{T} 72 \mathrm{f}$ \\
\hline \multicolumn{9}{|c|}{ Glicose $^{\mathrm{a}}$ (média \pm desvio-padrão) } \\
\hline $\mathrm{C}$ & $83,2 \pm 8,2$ & $66,8 \pm 10,6$ & $82,7 \pm 17,7$ & $78,2 \pm 10,3$ & $73,5 \pm 9,4$ & $73,3 \pm 2,5$ & $78,3 \pm 6,5$ & $77,4 \pm 10,3$ \\
\hline E8 & $87,8 \pm 9,8$ & $173,1 \pm 25,7$ & $155,7 \pm 77,2$ & $147,4 \pm 56,4$ & $178,1 \pm 34,2$ & $118,6 \pm 26,7$ & $97,0 \pm 24,3$ & $71,3 \pm 11,7$ \\
\hline SE & $73,2 \pm 10,4$ & $155,4 \pm 50,6$ & $131,8 \pm 62,9$ & $103,8 \pm 43,6$ & $84,7 \pm 9,0$ & $91,7 \pm 18,3$ & $73,2 \pm 6,1$ & $77,4 \pm 10,2$ \\
\hline \multirow[t]{2}{*}{ RL } & $79,7 \pm 5,1$ & $177,0 \pm 37,2$ & $130,6 \pm 39,1$ & $116,9 \pm 46,0$ & $97,7 \pm 38,6$ & $80,2 \pm 12,0$ & $74,7 \pm 5,3$ & $85,0 \pm 13,5$ \\
\hline & $\mathrm{C}=\mathrm{E} 8=\mathrm{SE}=\mathrm{RL}$ & $\mathrm{E} 8=\mathrm{SE}=\mathrm{RL}>\mathrm{C}$ & $\mathrm{E} 8=\mathrm{SE}=\mathrm{RL}>\mathrm{C}$ & $\mathrm{E} 8>\mathrm{SE}=\mathrm{C}$ & $\mathrm{E} 8>\mathrm{C}=\mathrm{SE}=\mathrm{RL}$ & $\mathrm{E} 8>\mathrm{C}$ & $\mathrm{C}=\mathrm{E} 8=\mathrm{SE}=\mathrm{RL}$ & $\mathrm{C}=\mathrm{E} 8=\mathrm{SE}=\mathrm{RL}$ \\
\hline \multicolumn{9}{|c|}{ Uréia $^{\text {a }}$ (média \pm desvio-padrão) } \\
\hline $\mathrm{C}$ & $21,2 \pm 3,4$ & $21,5 \pm 4,7$ & $21,4 \pm 3,2$ & $21,8 \pm 3,5$ & $25,4 \pm 1,9$ & $21,0 \pm 2,1$ & $19,2 \pm 3,1$ & $20,7 \pm 2,9$ \\
\hline E8 & $22,4 \pm 7,8$ & $39,2 \pm 14,6$ & $57,0 \pm 18,9$ & $97,9 \pm 25,8$ & $93,2 \pm 30,0$ & $66,7 \pm 16,9$ & $26,7 \pm 13,2$ & $21,1 \pm 5,6$ \\
\hline $\mathrm{SE}$ & $21,8 \pm 8,9$ & $41,0 \pm 11,0$ & $65,7 \pm 21,2$ & $93,8 \pm 16,8$ & $82,5 \pm 14,2$ & $101,9 \pm 6,0$ & $79,3 \pm 29,1$ & $66,3 \pm 42,1$ \\
\hline \multirow[t]{2}{*}{ RL } & $23,2 \pm 3,5$ & $36,2 \pm 9,5$ & $51,0 \pm 14,7$ & $73,9 \pm 42,8$ & $52,7 \pm 35,5$ & $40,1 \pm 18,3$ & $23,9 \pm 13,8$ & $25,5 \pm 10,2$ \\
\hline & $\mathrm{C}=\mathrm{E} 8=\mathrm{SE}=\mathrm{RL}$ & $\mathrm{C}=\mathrm{E} 8=\mathrm{SE}=\mathrm{RL}$ & $\mathrm{E} 8=\mathrm{SE}=\mathrm{RL}>\mathrm{C}$ & $\mathrm{E} 8=\mathrm{SE}=\mathrm{RL}>\mathrm{C}$ & $\mathrm{E} 8=\mathrm{SE}>\mathrm{RL}>\mathrm{C}$ & $\mathrm{SE}>\mathrm{E} 8>\mathrm{RL}=\mathrm{C}$ & $\mathrm{SE}>\mathrm{C}=\mathrm{E} 8=\mathrm{RL}$ & $\mathrm{SE}>\mathrm{C}=\mathrm{E} 8=\mathrm{RL}$ \\
\hline \multicolumn{9}{|c|}{ Creatinina $^{\mathrm{a}}$ (média \pm desvio-padrão) } \\
\hline $\mathrm{C}$ & $0,70 \pm 0,19$ & $0,68 \pm 0,16$ & $0,70 \pm 0,12$ & $0,70 \pm 0,14$ & $0,82 \pm 0,12$ & $0,71 \pm 0,14$ & $0,68 \pm 0,13$ & $0,74 \pm 0,10$ \\
\hline E8 & $0,73 \pm 0,23$ & $0,80 \pm 0,28$ & $1,04 \pm 0,26$ & $3,39 \pm 0,61$ & $2,66 \pm 0,90$ & $1,74 \pm 0,67$ & $1,01 \pm 0,28$ & $0,89 \pm 0,10$ \\
\hline $\mathrm{SE}$ & $0,59 \pm 0,11$ & $0,73 \pm 0,17$ & $0,94 \pm 0,41$ & $2,35 \pm 1,22$ & $1,63 \pm 0,81$ & $2,24 \pm 0,87$ & $2,22 \pm 1,09$ & $2,91 \pm 1,62$ \\
\hline \multirow[t]{2}{*}{ RL } & $0,67 \pm 0,12$ & $0,75 \pm 0,13$ & $1,08 \pm 0,50$ & $2,14 \pm 1,58$ & $1,26 \pm 0,89$ & $1,04 \pm 0,53$ & $0,95 \pm 0,50$ & $0,88 \pm 0,22$ \\
\hline & $\mathrm{C}=\mathrm{E} 8=\mathrm{SE}=\mathrm{RL}$ & $\mathrm{C}=\mathrm{E} 8=\mathrm{SE}=\mathrm{RL}$ & $\mathrm{C}=\mathrm{E} 8=\mathrm{SE}=\mathrm{RL}$ & $\mathrm{E} 8>\mathrm{SE}=\mathrm{RL}>\mathrm{C}$ & E8 $>$ SE,RL,C SE $>$ C & $\mathrm{SE}>\mathrm{RL}, \mathrm{C} \mathrm{E} 8>\mathrm{C}$ & $\mathrm{SE}>\mathrm{C}=\mathrm{E} 8=\mathrm{RL}$ & $\mathrm{SE}>\mathrm{C}=\mathrm{E} 8=\mathrm{RL}$ \\
\hline \multicolumn{9}{|c|}{ Lactato $^{a}$ (média \pm desvio-padrão) } \\
\hline $\mathrm{C}$ & $0,67 \pm 0,18$ & $0,36 \pm 0,21$ & $0,61 \pm 0,17$ & $0,57 \pm 0,14$ & $0,51 \pm 0,21$ & $0,67 \pm 0,12$ & $0,34 \pm 0,10$ & $0,55 \pm 0,10$ \\
\hline E8 & $0,73 \pm 0,26$ & $1,33 \pm 0,40$ & $1,61 \pm 0,52$ & $2,21 \pm 0,68$ & $1,80 \pm 0,52$ & $1,76 \pm 0,66$ & $1,35 \pm 0,82$ & $1,20 \pm 0,28$ \\
\hline $\mathrm{SE}$ & $0,76 \pm 0,18$ & $2,26 \pm 1,00$ & $2,29 \pm 0,80$ & $2,65 \pm 1,22$ & $3,11 \pm 0,86$ & $1,96 \pm 0,85$ & $1,54 \pm 0,23$ & $1,56 \pm 0,68$ \\
\hline \multirow[t]{2}{*}{$\mathrm{RL}$} & $0,71 \pm 0,18$ & $1,55 \pm 0,65$ & $1,46 \pm 0,45$ & $1,92 \pm 0,58$ & $2,60 \pm 1,01$ & $1,20 \pm 0,53$ & $1,01 \pm 0,40$ & $1,03 \pm 0,30$ \\
\hline & $\mathrm{C}=\mathrm{E} 8=\mathrm{SE}=\mathrm{RL}$ & $\mathrm{SE}>\mathrm{E} 8, \mathrm{C}$ RL $>\mathrm{C}$ & $\mathrm{SE}>\mathrm{RL}, \mathrm{C}$ E8 $>\mathrm{C}$ & $\mathrm{E} 8=\mathrm{SE}=\mathrm{RL}>\mathrm{C}$ & $\mathrm{SE}=\mathrm{RL}>\mathrm{E} 8>\mathrm{C}$ & $\mathrm{E} 8=\mathrm{SE}>\mathrm{C}$ & $\mathrm{E} 8=\mathrm{SE}>\mathrm{C}$ & $\mathrm{SE}>\mathrm{C}$ \\
\hline \multicolumn{9}{|c|}{ Magnésio total ${ }^{\text {a }}$ (média \pm desvio-padrão) } \\
\hline $\mathrm{C}$ & $1,0 \pm 0,1$ & $1,0 \pm 0,1$ & $1,0 \pm 0,1$ & $1,2 \pm 0,3$ & $1,1 \pm 0,1$ & $1,0 \pm 0,1$ & $1,0 \pm 0,1$ & $1,1 \pm 0,1$ \\
\hline E8 & $1,5 \pm 0,4$ & $1,8 \pm 0,4$ & $2,2 \pm 0,2$ & $2,3 \pm 0,2$ & $1,9 \pm 0,5$ & $1,3 \pm 0,2$ & $0,7 \pm 0,2$ & $1,0 \pm 0,3$ \\
\hline SE & $1,3 \pm 0,3$ & $1,9 \pm 0,4$ & $1,6 \pm 0,5$ & $1,4 \pm 0,5$ & $1,3 \pm 0,3$ & $1,3 \pm 0,4$ & $1,2 \pm 0,1$ & $1,2 \pm 0,3$ \\
\hline \multirow[t]{2}{*}{$\mathrm{RL}$} & $1,5 \pm 0,2$ & $1,8 \pm 0,3$ & $1,9 \pm 0,6$ & $1,9 \pm 0,7$ & $1,2 \pm 0,4$ & $1,4 \pm 0,3$ & $1,3 \pm 0,3$ & $1,3 \pm 0,1$ \\
\hline & $\mathrm{E} 8=\mathrm{RL}>\mathrm{C}$ & $\mathrm{E} 8=\mathrm{SE}=\mathrm{RL}>\mathrm{C}$ & E8 $>$ SE, C RL $>C$ & $\mathrm{E} 8>\mathrm{SE}, \mathrm{C}$ RL $>\mathrm{C} ; \mathrm{SE}>\mathrm{C}$ & $\mathrm{E} 8>\mathrm{C}=\mathrm{SE}=\mathrm{RL}$ & $\mathrm{RL}>\mathrm{C}$ & $\mathrm{RL}>\mathrm{E} 8$ & $\mathrm{C}=\mathrm{E} 8=\mathrm{SE}=\mathrm{RL}$ \\
\hline
\end{tabular}

T0i-T72i: período de indução de compactação.

T12t-T48t: período de tratamento.

T72t: período final de tratamento.

${ }^{a}$ Análise de variância (medidas repetidas).

$\mathrm{C}=$ controle; $\mathrm{E} 8=$ solução isotônica poliônica enteral $\left(\mathrm{NaCl} 5 \mathrm{~g}+\mathrm{KCl} 1 \mathrm{~g}+\mathrm{NaHCO}_{3} 4 \mathrm{~g}+\mathrm{glicose} 5 \mathrm{~g}-\mathrm{qsp} 1000 \mathrm{ml}\right)$, dose de $8 \mathrm{ml} / \mathrm{km} / \mathrm{h} / 48 \mathrm{~h} \mathrm{na}$ sonda nasogástrica; $\mathrm{SE}=$ solução isotônica intravenosa de Ringer lactato $(10 \mathrm{ml} / \mathrm{kg} / \mathrm{h} / 12 \mathrm{~h})$ associada à sene, dose de $20 \mathrm{mg} / \mathrm{kg}$; $\mathrm{RL}=$ solução isotônica de Ringer lactato $(16 \mathrm{ml} / \mathrm{kg} / \mathrm{h} / 12 \mathrm{~h})$.

Ocorreu aumento significativo (Tab. 2) na concentração da glicose nos grupos E8, SE e RL $(\mathrm{P}=0,0038)$ no período de indução da compactação (T0i a T72i). Esse aumento devese ao estresse que, via sistema neuroendócrino, aumenta a concentração do cortisol, que, por sua vez, aumenta a glicemia (Cunningham,
1993) associada à ação do amitraz, como citou Queiroz-Neto et al. (2000). A hiperglicemia, nos casos de cólica, é também mediada pela secreção de adrenalina em resposta à dor (Parry, 1987). Com o fim da fase de indução e, conseqüentemente, do estresse, a partir do T12t, os valores da glicemia dos grupos SE e RL 
declinaram gradualmente e no T72f apresentaram valores semelhantes aos do T0i. Ao contrário, nos animais do grupo $\mathrm{E} 8$, no $\mathrm{T} 12 \mathrm{t}$, os valores elevaram-se, atingindo valores semelhantes ao do T24i e T48i ( $\mathrm{P}=0,0001)$, ocasionados pela composição da solução enteral. No T24t e T48t, houve diminuição gradual da glicemia, apesar da administração da solução enteral. Nesses dois tempos, T24t e T48t, já haviam transcorrido 24 e 48 horas de fluidoterapia, respectivamente, determinando a recuperação da volemia nos animais, o que permitiu aos rins eliminarem o excesso de glicose pela urina e sua melhor utilização pelo fígado e músculos, como descreveram Kaneco et al. (1997).

$\mathrm{O}$ aumento gradual das concentrações séricas de uréia $(\mathrm{P}=0,0001)$ e creatinina $(\mathrm{P}=0,0001)$ durante a fase de indução da compactação foi ocasionado pela desidratação que levou à hipovolemia, a qual reduziu o fluxo sangüíneo renal e a taxa de filtração glomerular, prejudicando a excreção de uréia e creatinina, originando, assim, azotemia pré-renal (Kaneco et al., 1997). Pela Tab. 2 verifica-se que, a partir de T12t (início da fluidoterapia), começou a ocorrer declínio gradual dessas substâncias, atingindo no T72f valores semelhantes aos do T0i, com exceção do grupo SE. A concentração de uréia do grupo SE no T72f apresentou valores superiores aos de referência (10-24mg/dl; Kaneco et al., 1997). A concentração sérica de creatinina nesse grupo, a partir do T12t, começou a aumentar, atingindo no $\mathrm{T} 72 \mathrm{f}$ os maiores valores $(\mathrm{P}=0,0001)$, sugerindo que a fluidoterapia não foi adequada com relação ao volume e ao tempo para retirar esses animais do quadro de azotemia pré-renal.

O lactato aumentou durante a fase de indução da compactação (T0i a T72i) nos grupos E8, SE e RL $(\mathrm{P}=0,0001)$; esse aumento foi determinado pela desidratação. A hipovolemia decorrente da desidratação induz à baixa perfusão tecidual, resultando em limitado fornecimento de oxigênio aos tecidos e diminuição na excreção de íons $\mathrm{H}+$ pelos rins. A hipóxia tecidual aumenta a biossíntese do ácido láctico originário do metabolismo anaeróbico (glicólise), liberando-o mais rápido do que ele possa ser oxidado ou reconvertido em glicose ou glicogênio pelo fígado conforme considerou Gosset et al. (1987).
Após 12 horas de tratamento, os valores do lactato continuaram aumentando nos animais dos grupos $\mathrm{SE}$ e $\mathrm{RL}(\mathrm{P}=0,0001)$. Mesmo após a expansão do líquido extracelular, a célula ainda permanece com falta de líquido por determinado período e, no processo de reidratação, mesmo em quantidade menor, ela ainda continuará a produzir substâncias ácidas conseqüentes do metabolismo anaeróbico. Além disso, na correção dos desequilíbrios hidroeletrolíticos e ácidobase, ocorre o retorno do potássio para o interior das células e a saída de substâncias ácidas para o plasma. Observa-se que nesses grupos, a partir do T12t, os valores do lactato plasmático começaram a diminuir. No grupo E8, nas primeiras 12 horas de tratamento, os valores do lactato plasmático diminuíram, oposto ao ocorrido nos grupos $\mathrm{SE}$ e RL, indicando que o bicarbonato de sódio, presente na solução isotônica poliônica enteral ( $4 \mathrm{~g} /$ litro da solução), foi mais eficiente no tamponamento do lactato, pois sua ação alcalinizante é direta (Naylor et al., 1990).

Neste estudo, a concentração sérica de magnésio total aumentou durante a fase de indução da compactação nos grupos E8, SE e RL $(\mathrm{P}=0,0037)$. As causas foram a desidratação e o desequilíbrio ácido-base presentes. O aumento do magnésio sérico nos grupos E8, SE e RL durante a fase de indução difere dos resultados de Costa et al. (2002), que encontraram hipomagnesemia em eqüinos com obstrução simples do intestino grosso, freqüentemente acompanhada por outras anormalidades eletrolíticas. GarciaLopez et al. (2001) avaliaram eqüinos com afecções estrangulantes e não estrangulantes do intestino grosso, encontrando hipomagnesemia apenas nos animais com afecções estrangulantes e íleo. Concluíram que o magnésio livre foi mais eficiente do que o magnésio total na detecção de alteração do seu teor. A partir do T72i, os valores começaram a diminuir no grupo $\mathrm{SE}$ e, a partir de T12t, nos grupos E8 e RL, alcançando no T72f valores semelhantes aos do T0i. A correção dos desequilíbrios hidroeletrolíticos e a do ácidobase foram os fatores determinantes para 0 ocorrido. No presente estudo verificou-se hipomagnesemia apenas no grupo $\mathrm{E} 8(\mathrm{P}=0,0001)$ no T48t, o que pode ter sido ocasionado pela 
fluidoterapia prolongada, 48 horas, com solução sem magnésio, confirmando as citações de DiPalma (1990) e Dart et al. (1992).

\section{CONCLUSÕES}

A fluidoterapia intravenosa com solução de Ringer lactado foi o tratamento mais eficiente para correção dos valores bioquímicos em eqüinos com compactação induzida do cólon maior, seguido pela fluidoterapia enteral com solução isotônica poliônica. A fluidoterapia intravenosa associada à sene foi o tratamento menos eficiente na normalização das variáveis bioquímicas.

\section{REFERÊNCIAS BIBLIOGRÁFICAS}

CONOVER, W.J. Practical nonparametric statistics. New York: John Wiley \& Sons, 1980. 493p.

COSTA, L.R.R.; EADES, S.C.; TULLEY, R.T. et al. Plasma magnesium and other electrolyte abnormalities in horses affected with gastrointestinal tract disease. In: INTERNATIONAL EQUINE COLIC RESEARCH SYMPOSIUM, 7. Manchester, 2002. p.95-96.

CUNNINGHAM, J.G. Tratado de fisiologia veterinária. Rio de Janeiro: Guanabara Koogan, 1993. 454p.

DABAREINER, R. M.; WHITE, N. A. Large colon impaction: 147 cases (1985-1991). J. Am. Vet. Assoc., v.206, p.679-685, 1995.

DART, A.J.; SNYDER, J.R.; SPIER, S.J. et al. Ionized calcium concentration in horses with surgically managed gastrointestinal disease: 147 cases (1988-1990). J. Am. Vet. Assoc., v.191, p.849-854, 1992.

DiBARTOLA, S.P. Disorders of sodium and water: hypernatremia and hyponatremia. In: _. Fluid therapy in small animal practice. Philadelphia: Saunders, 1992. p.57-88.

DiPALMA, J.R. Magnesium replacement therapy. Am. Fam. Physician, v.42, p.173-176, 1990.

DORAN, R. Field management of simple intestinal obstruction in horses. Comp. Cont. Educ. Pract. Vet., v.15, p.463-471, 1993.

FRANZ, D.E. The senna drug and its chemistry. Pharmacology, v.47, suppl. 1, p.2-6, 1993.

FREEMAN, D.E.; FERRANTE, P.L.; PALMER, J.E. Comparison of the effects of intragastric infusions of equal volumes of water, dioctyl sodium sulfoccinate, and magnesium sulfate on fecal composition and output in clinically normal horses. Am. J. Vet. Res., v.53, p.13471353, 1992.

FREESTONE, J.F.; CARLSON, G.P.; HARROLD, D.R. et al. Furosemide and sodium bicarbonate-induced alkalosis in the horse and response to oral $\mathrm{KCl}$ or $\mathrm{NaCl}$ therapy. Am. J. Vet. Res., v.50, p.1334-1339, 1989.
GARCIA-LOPEZ, J.M.; PROVOST, P.J.; RUSH, J.E. et al. Prevalence and prognostic importance of hipomagnesemia and hypocalcemia in horses that have colic surgery. Am. J. Vet. Res., v.62, p.7-12, 2001.

GENETZKY, R.M.; LOPARCO, F.V.; LEDET, A.E. Clinical pathologic alterations in horse during a water deprivation test. Am. J. Vet. Res., v.48, p.1007-1011, 1987.

GENNARI, F.J. Serum osmolality: uses and limitations. N. Engl. J. Med., v.12, p.102-105, 1984.

GOSSET, K.A.; CLEGHORN, B.S.; ADAMS, R. et al. Contribuition of whole blood L-lactate, pyruvate, Dlactate, acetoacetate, and 3-hydroxybutirate concentrations to the plasma anion gap in horses with intestinal disordens. Am. J. Vet. Res., v.48, p.72-75, 1987.

JOHNSON, P.J. Electrolyte and acid-base disturbances in the horse. Vet. Clin. North Am.: Equine Pract., v.11, p.491-514, 1995.

KANECO, J.R.; HARVEY, J.W.; BRUSS, M.L. Clinical biochemistry of domestic animal. 5.ed. San Diego: Academic, 1997. 932p.

KASARI, T.R. Metabolic acidosis in calves. Vet. Clin. North Am.: Food Anim. Pract., v.15, p.473-486, 1999.

LEMLI, J. Metabolism of sennosides - An overview. Pharmacology, v.346, suppl 1, p.126-128, 1988

NAYLOR, J.M.; PETRIE, L.; RODRIGUEZ, M.I. A comparasion of three oral electrolyte solutions in the treatment of diarrheic calves. Can. Vet. J., v.1, p.750-753, 1990.

PARRY, B.W. Use clinical pathology in evaluation of horses with colic. Vet. Clin. North Am.: Equine Pract., v.3, p.520-541, 1987.

RIBEIRO FILHO, J.D.; ALVES, G.E.S. Compactação no intestino grosso de eqüinos: estudo comparativo de três protocolos de indução. Arq. Bras. Med. Vet. Zootec., v.54, p.370-375, 2002.

SPEIRS, V.C. The alimentary tract. In: . Clinical examination of horses. Philadelphia: Saunders, 1997. p.261-298.

SULLINS K.E. Disease of the large colon. In: WHITE, N.A. Equine acute abdomen. Philadelphia: Lea \& Febiger, 1990. p.375-391.

USER's guide: statistics, version 5. Cary, NC: SAS Institute, 1985.

WHITE, N.A. Epidemiology and etiology of colic. In:_. Equine acute abdomen. Phidelphia: Lea \& Febiger, 1990. p.50-64.

WHITE, N.A.; DABAREINER, R.M. Treatment of impaction colonic. Vet. Clin. North Am.: Equine Pract., v.13, p.243-259, 1997.

YAGI, T.; MIYAWAKI, Y.; NISHIKAWA, A. et al. Suppression of the purgative action of rheinanthrone the active metabolite of sennosides A and B by indomethacin in rats. J. Pharm. Pharmacol., v.43, p.307-310, 1991. 